\title{
Explicit solutions to the Korteweg-de Vries equation on the half line
}

\author{
Tuncay Aktosun ${ }^{1}$ and Cornelis van der $\mathrm{Mee}^{2}$ \\ ${ }^{1}$ Department of Mathematics, University of Texas at Arlington, Arlington, TX 76019-0408, USA \\ ${ }^{2}$ Dipartimento di Matematica e Informatica, Università di Cagliari, Viale Merello 92, \\ 09123 Cagliari, Italy
}

Received 30 May 2006, in final form 4 October 2006

Published 20 October 2006

Online at stacks.iop.org/IP/22/2165

\begin{abstract}
Certain explicit solutions to the Korteweg-de Vries equation in the first quadrant of the $x t$-plane are presented. Such solutions involve algebraic combinations of truly elementary functions, and their initial values correspond to rational reflection coefficients in the associated Schrödinger equation. In the reflectionless case such solutions reduce to pure $N$-soliton solutions. An illustrative example is provided.
\end{abstract}

\section{Introduction}

Consider the celebrated Korteweg-de Vries (KdV) equation

$$
\frac{\partial u}{\partial t}+\eta \frac{\partial u}{\partial x}-6 u \frac{\partial u}{\partial x}+\frac{\partial^{3} u}{\partial x^{3}}=0
$$

where $x$ and $t$ denote the spatial and temporal variables, respectively, and $\eta$ is a nonnegative constant $[1,2]$ that can be chosen as 0 or 1 . The $\mathrm{KdV}$ equation is used to model [3-5] the propagation of water waves in long, narrow, shallow channels; it also arises in other areas such as hydromagnetic waves in a cold plasma, ion-acoustic waves and acoustic waves in harmonic crystals.

The KdV equation is one of the most well-known and most widely analysed nonlinear partial differential equations. It has many remarkable aspects $[4,5]$, for example, it possesses travelling wave solutions known as solitons. The numerical studies on the KdV equation by Zabusky and Kruskal [3] led to the discovery of multi-soliton solutions, where the individual solitons interact nonlinearly at close distance and then move away from each other without changing their shapes. In their celebrated paper [6] Gardner, Greene, Kruskal and Miura showed that the initial-value problem for the $\mathrm{KdV}$ equation can be solved via the 'inverse scattering transform' associated with the Schrödinger equation. This led to the discovery that certain nonlinear partial differential equations are 'completely integrable': they can be solved via an inverse scattering transform and they have some interesting common properties such as possessing soliton solutions, Lax pairs and infinitely many conserved quantities. 
A pure $N$-soliton solution to the $\mathrm{KdV}$ equation can be written explicitly as $[4,5,7,8]$

$$
u(x, t)=-2 \frac{\partial^{2} \log (\operatorname{det} \Gamma(x ; t))}{\partial x^{2}},
$$

where $\Gamma(x ; t)$ is the $N \times N$ matrix whose $(j, l)$ entry is given by [9]

$$
\Gamma_{j l}=\delta_{j l}+\frac{c_{j} \mathrm{e}^{-2 \kappa_{j} x+8 \kappa_{j}^{3} t+2 \eta \kappa_{j} t}}{\kappa_{j}+\kappa_{l}}, \quad 1 \leqslant j, l \leqslant N,
$$

with $\delta_{j l}$ denoting the Kronecker delta, $\kappa_{j}$ are $N$ distinct positive constants corresponding to the bound states of $u(x, 0)$ and $c_{j}$ are $N$ positive constants known as the bound-state norming constants. Pure soliton solutions are trivial in the sense that the potential $u(x, 0)$ corresponds [3-5] to a zero reflection coefficient in the Schrödinger equation. There are many important ways explicit solutions to the $\mathrm{KdV}$ equation may help us to understand nonlinearity better. For example, it is of great importance $[1,10]$ to determine function spaces containing the initial data $u(x, 0)$ so that $u(x, t)$ is globally well behaved (i.e., does not blow up during $t \in[0,+\infty)$ ) or only locally well behaved (i.e., remains finite during $t \in[0, \tau)$ and blows up at some finite time $\tau$ ), and explicit solutions may help us to understand the global or local well-posedness of initial-value problems for the $\mathrm{KdV}$ equation. Explicit solutions may contribute to the development or improvement of numerical methods for (1.1) and they may also be useful to check the accuracy of existing numerical methods.

In this paper, we present a method leading to certain explicit solutions to the KdV equation in the first quadrant of the $x t$-plane. Let us emphasize that our aim is not to solve the initialboundary-value problem for (1.1) in the quarter plane. Instead, we are interested in producing some explicit solutions to (1.1) in terms of truly elementary functions. We produce certain explicit solutions to (1.1) with the form (1.2), where $\Gamma(x ; t)$ is the matrix appearing in (4.1). From (4.1) it is seen that $\Gamma(x ; t)$ can be constructed explicitly by specifying a constant $P \times P$ matrix $A$, a constant $P$-row vector $C$ and a constant $P$-column vector $B$. We also show that such solutions can equivalently be written as in (4.9). In fact, it is straightforward (but tedious) to verify that the right-hand side in (4.9) is a (formal) solution to (1.1) no matter how $A, B$ and $C$ are chosen. However, as seen from (4.1), an arbitrary choice for $A$ may not guarantee the convergence of the integral in (4.1); even if $\Gamma(x ; t)$ obtained from (4.1) exists by choosing $A$ appropriately, that particular choice for $A$ and arbitrary choices for $B$ and $C$ may not assure the existence of $\Gamma(x ; t)^{-1}$ appearing in (4.9) for $x \in[0,+\infty)$ and $t \in[0, \tau)$ for some $\tau>0$ or $\tau=+\infty$. One of our tasks in this paper is to indicate how we may choose $A, B, C$ in order to assure the existence of $\Gamma(x ; t)$ and the positivity of its determinant for all $x \in[0,+\infty)$ and $t \in[0, \tau)$, which in turn assures the existence and well-posedness of the solution $u(x, t)$ given in (4.9).

One set of possible choices for $A, B, C$ corresponds to the initial values $u(x, 0)$ for $x \in[0,+\infty)$ in such a way that $u(x, 0)$ becomes the potential belonging to a certain class in the one-dimensional Schrödinger equation. For example, $u(x, 0)$ may be viewed as a fragment of a real-valued and integrable potential, which has a finite first moment and which corresponds to a rational reflection coefficient. Other choices may be possible, e.g., we may further require that the corresponding one-dimensional potential $u(x, 0)$ vanishes identically for $x<0$. In fact, in section 3 we outline how $A, B, C$ can be explicitly constructed from such a potential. All such choices guarantee the existence of the integral in (4.1) for all $x \in[0,+\infty)$ and each fixed $t$. This is because such choices, as a result of using (3.2), assure that each eigenvalue of $A$ has a positive real part. Hence, the matrix $\Gamma(x ; t)$ defined in $(4.1)$ exists for all $x \in[0,+\infty)$ and each fixed $t$, its determinant $\operatorname{det} \Gamma(x ; t)$ is continuous in $t$ for every $x \in[0,+\infty)$, and also det $\Gamma(x ; t) \rightarrow 1$ as $x \rightarrow+\infty$ for each fixed $t$. Furthermore, for such choices it is already known [11] that the resulting $u(x, 0)$ is analytic in $x$ and det $\Gamma(x ; 0)>0$ for all $x \in[0,+\infty)$. 
Consequently, we have $\operatorname{det} \Gamma(x ; t)>0$ for all $x \in[0,+\infty)$ and $t \in[0, \tau)$ for some positive $\tau$. It is remarkable that, for certain choices of $A, B, C$, we can have $\tau=+\infty$, as we see from the example in section 5 .

As seen from the analysis in section 3 , in case the relevant reflection coefficient is zero, our solution $u(x, t)$ reduces to the pure $N$-soliton solution given in (1.2)-(1.3). This is equivalent to choosing in (4.1) and (4.9) the matrix $A$ as the $N \times N$ diagonal matrix with $\kappa_{j}$ appearing in the $(j, j)$ entry, $B$ as the column $N$-vector having the number 1 in each entry and $C$ as the row $N$-vector with $c_{j}$ appearing in the $j$ th entry.

This paper is organized as follows. In section 2, we mention some of the other methods to solve the $\mathrm{KdV}$ equation and give a brief comparison. In section 3, we provide a physical motivation for the derivation of our solutions and show how they may be related to some scattering data. In section 4, we show how our solutions can be obtained by solving the Marchenko integral equation. Finally, in section 5 we present an example to illustrate our method.

\section{Some other methods for the KdV equation}

As seen from section 3, our method is based on using the inverse scattering transform, exploiting the degeneracy of the kernel of the Marchenko integral equation as indicated in (4.7) and solving the Marchenko equation (4.4) algebraically. There are also methods to solve the $\mathrm{KdV}$ equation without using the inverse scattering transform; for example, the technique $[12,13]$ based on using the Bäcklund transformation, the technique $[8,14]$ using the Darboux-Crum transformation, the Wronskian techniques and their generalizations [7, 15-18] and the Hirota method [19]. Such methods are also used to produce certain exact solutions to the KdV equation. The idea behind the methods using the transformations of Bäcklund and Darboux-Crum is to obtain new solutions to the KdV equation from other previously known solutions. The basic idea behind the Wronskian methods and the Hirota method is to represent the solution to the $\mathrm{KdV}$ equation in a particular form so that certain functions in the representation satisfy certain linear differential equations even though the solution itself satisfies a nonlinear differential equation. The explicit solutions produced by our method have the same representation (1.2) or (4.2) as in the Wronskian methods; however, our matrix $\Gamma(x ; t)$ (or a part of it) does not necessarily satisfy a linear partial differential equation as expected in the Wronskian methods. In the method based on the Darboux-Crum transformation, the solution to the $\mathrm{KdV}$ equation has the same representation as in (1.2) or (4.2), provided the initial solution is chosen as zero; there is certainly some connection between that method and our method because they both yield the $N$-soliton solution in the easiest case; however, any possible connection in the more general case is not clear at the moment and requires a detailed analysis, which we plan to do in the future. For the time being, we only emphasize that our exact solutions satisfy the half-line $\mathrm{KdV}$ equation with the drift term $\eta u_{x}$ where we can choose $\eta=0$ or $\eta>0$ at will, they include some global-in-time solutions as well as some local-in-time solutions, and they are algebraic combinations of truly elementary functions. One advantage of our method is that it can be generalized to obtain certain explicit solutions to the matrix KdV equation as well as to the scalar and matrix nonlinear Schrödinger equations.

Some other explicit solutions to the KdV equation known in the literature include algebraic solitons [20-22], rational solutions [22, 23], various singular solutions [24-26] such as positons and negatons, solutions [22] to the periodic and other KdV equations, solutions [27] that are not quite as explicit but expressed in terms of certain projection operators and various other solutions $[28,29]$. It is already known that some rational solutions can be obtained by letting the bound-state energies go to zero in the $N$-soliton solutions. We plan to do in the future 
a detailed comparison between our solutions (and their possible generalizations) and exact solutions obtained by other methods. Some generalizations of our solutions might be obtained by letting the dimension of the matrix $A$ in (3.3) go to infinity, by choosing the entries of $A$ and $C$ given in (3.3) in some particular way or by letting some entries go to certain limits such as zero and by analysing the singularities encountered at $t=\tau$.

\section{Some possible choices for $A, B, C$}

In this section, we indicate a possible set of choices for $A, B, C$ appearing in $\Gamma(x ; t)$ of $(4.1)$ so that the resulting function $u(x, t)$ given in (1.2) or (4.2), or equivalently that in (4.9), is an explicit solution to (1.1) for all $x \in[0,+\infty)$ and $t \in[0, \tau)$ with some positive $\tau$.

Starting with the initial value $u(x, 0)$ with $x>0$, we extend it to the whole line by choosing $u(x, 0) \equiv 0$ for $x<0$ and we uniquely determine the corresponding scattering data $\left\{R,\left\{\kappa_{j}\right\},\left\{c_{j}\right\}\right\}$. Here, $R(k)$ is the corresponding right reflection coefficient [30-34], the set of constants $\kappa_{j}$ with $0<\kappa_{1}<\cdots<\kappa_{N}$ corresponds to the bound states associated with the full-line potential $u(x, 0)$ and the set of constants $c_{j}$ corresponds to the associated boundstate norming constants. The construction of $\left\{R,\left\{\kappa_{j}\right\},\left\{c_{j}\right\}\right\}$ can be accomplished through the following steps:

(a) Given $u(x, 0)$ for $x \in[0,+\infty)$, uniquely determine the corresponding Jost solution $f_{\mathrm{r}}(k, x)$ from the right by solving the initial-value problem for the half-line Schrödinger equation:

$$
\frac{\mathrm{d}^{2} f_{\mathrm{r}}}{\mathrm{d} x^{2}}+k^{2} f_{\mathrm{r}}=u(x, 0) f_{\mathrm{r}} ; \quad f_{\mathrm{r}}(k, 0)=1, \quad \frac{\mathrm{d} f_{\mathrm{r}}(k, 0)}{\mathrm{d} x}=-\mathrm{i} k .
$$

(b) Recover the corresponding right reflection coefficient $R$ and the transmission coefficient $T$ with the help of the asymptotics [30-34] of $f_{\mathrm{r}}$ as $x \rightarrow+\infty$, namely by using

$$
f_{\mathrm{r}}(k, x)=\frac{1}{T(k)} \mathrm{e}^{-\mathrm{i} k x}+\frac{R(k)}{T(k)} \mathrm{e}^{\mathrm{i} k x}+o(1), \quad x \rightarrow+\infty .
$$

It is known [30-34] that $T$ is related to $R$ via

$$
T(k)=\prod_{j=1}^{N}\left(\frac{k+\mathrm{i} \kappa_{j}}{k-\mathrm{i} \kappa_{j}}\right) \exp \left(\frac{1}{2 \pi \mathrm{i}} \int_{-\infty}^{\infty} \mathrm{d} s \frac{\log \left(1-|R(s)|^{2}\right)}{s-k-\mathrm{i} 0^{+}}\right), \quad k \in \overline{\mathbf{C}^{+}},
$$

where $\overline{\mathbf{C}^{+}}:=\mathbf{C}^{+} \cup \mathbf{R}, \mathbf{C}^{+}$is the upper half complex plane and $0^{+}$indicates that the limit from $\mathbf{C}^{+}$should be used to evaluate $T(k)$ for real $k$ values.

(c) Construct the set $\left\{\kappa_{j}\right\}_{j=1}^{N}$ by using (3.1).

(d) Construct the set of positive constants $\left\{c_{j}\right\}_{j=1}^{N}$ by using [30]

$$
c_{j}=-\left[\operatorname{Res}\left(T, \mathrm{i} \kappa_{j}\right)\right]^{2}\left[\frac{1}{2 \kappa_{j}}+\int_{0}^{\infty} \mathrm{d} x f_{\mathrm{r}}\left(\mathrm{i} \kappa_{j}, x\right)^{2}\right],
$$

where the purely imaginary constant $\operatorname{Res}\left(T, \mathrm{i} \kappa_{j}\right)$ denotes the residue of $T$ at $k=\mathrm{i} \kappa_{j}$.

Having constructed $R$ which is a rational function of $k$, we determine all its poles in $\mathbf{C}^{+}$ and the coefficients in the partial fraction expansion of $R$ at such poles. It is known [30-34] that $R\left(-k^{*}\right)=R(k)^{*}$ with the asterisk denoting complex conjugation, and hence such poles are either located on the positive imaginary axis $\mathbf{I}^{+}$or they occur in pairs symmetrically located with respect to $\mathbf{I}^{+}$. Let us use $M$ to denote the number of poles in $\mathbf{C}^{+}$without counting the multiplicities, and let us order them in such a way that the first $n$ pairs are located off $\mathbf{I}^{+}$at $k= \pm \alpha_{j}+\mathrm{i} \beta_{j}$ with $\alpha_{j}>0$ and $0<\beta_{1} \leqslant \cdots \leqslant \beta_{n}$; in case several distinct $\alpha_{j}$ values 
correspond to the same $\beta_{j}$, we can further arrange $\alpha_{j}$ in increasing order. We choose our notation so that the remaining $M-2 n$ poles occur at $k=\mathrm{i} \omega_{j}$ on $\mathbf{I}^{+}$with $0<\omega_{2 n+1}<\cdots<\omega_{M}$. We let $m_{j}$ indicate the multiplicity of the $j$ th pole.

Let $\Pi R$ denote the part of the partial fraction expansion of $R$ containing only the poles in $\mathbf{C}^{+}$. We have

$\Pi R(k)=\sum_{j=1}^{n} \sum_{s=1}^{m_{j}}\left[\frac{(-\mathrm{i})^{s}\left(\epsilon_{j s}+\mathrm{i} \gamma_{j s}\right)}{\left(k-\mathrm{i} \beta_{j}-\alpha_{j}\right)^{s}}+\frac{(-\mathrm{i})^{s}\left(\epsilon_{j s}-\mathrm{i} \gamma_{j s}\right)}{\left(k-\mathrm{i} \beta_{j}+\alpha_{j}\right)^{s}}\right]+\sum_{j=2 n+1}^{M} \sum_{s=1}^{m_{j}} \frac{(-\mathrm{i})^{s} r_{j s}}{\left(k-\mathrm{i} \omega_{j}\right)^{s}}$.

As a result of $R\left(-k^{*}\right)=R(k)^{*}$, the constants $\epsilon_{j s}, \gamma_{j s}$ and $r_{j s}$ appearing in (3.2) are all real; in fact, we have

$$
\begin{array}{ll}
\epsilon_{j s}+\mathrm{i} \gamma_{j s}=\left.\frac{\mathrm{i}^{s}}{\left(m_{j}-s\right) !} \frac{\mathrm{d}^{m_{j}-s}}{\mathrm{~d} k^{m_{j}-s}}\left[R(k)\left(k-\alpha_{j}-\mathrm{i} \beta_{j}\right)^{m_{j}}\right]\right|_{k=\alpha_{j}+\mathrm{i} \beta_{j}}, & j=1, \ldots, n, \\
r_{j s}=\left.\frac{\mathrm{i}^{s}}{\left(m_{j}-s\right) !} \frac{\mathrm{d}^{m_{j}-s}}{\mathrm{~d} k^{m_{j}-s}}\left[R(k)\left(k-\mathrm{i} \omega_{j}\right)^{m_{j}}\right]\right|_{k=\mathrm{i} \omega_{j}}, & j=2 n+1, \ldots, M .
\end{array}
$$

For $j=1, \ldots, n$, let us define $C_{j}:=2\left[\begin{array}{lllll}\gamma_{j m_{j}} & \epsilon_{j m_{j}} & \cdots & \gamma_{j 1} & \epsilon_{j 1}\end{array}\right]$ and

$$
A_{j}:=\left[\begin{array}{cccccc}
\Lambda_{j} & -I_{2} & 0 & \ldots & 0 & 0 \\
0 & \Lambda_{j} & -I_{2} & \ldots & 0 & 0 \\
0 & 0 & \Lambda_{j} & \ldots & 0 & 0 \\
\vdots & \vdots & \vdots & \ddots & \vdots & \vdots \\
0 & 0 & 0 & \ldots & \Lambda_{j} & -I_{2} \\
0 & 0 & 0 & \ldots & 0 & \Lambda_{j}
\end{array}\right], \quad B_{j}:=\left[\begin{array}{c}
0 \\
\vdots \\
0 \\
1
\end{array}\right]
$$

where $I_{2}$ denotes the $2 \times 2$ unit matrix, each column vector $B_{j}$ has $2 m_{j}$ components, each $A_{j}$ has size $2 m_{j} \times 2 m_{j}$ and each $2 \times 2$ matrix $\Lambda_{j}$ is defined as

$$
\Lambda_{j}:=\left[\begin{array}{cc}
\beta_{j} & \alpha_{j} \\
-\alpha_{j} & \beta_{j}
\end{array}\right]
$$

Similarly, for $j=2 n+1, \ldots, M$, let

$$
A_{j}:=\left[\begin{array}{cccccc}
\omega_{j} & -1 & 0 & \ldots & 0 & 0 \\
0 & \omega_{j} & -1 & \ldots & 0 & 0 \\
0 & 0 & \omega_{j} & \ldots & 0 & 0 \\
\vdots & \vdots & \vdots & \ddots & \vdots & \vdots \\
0 & 0 & 0 & \ldots & \omega_{j} & -1 \\
0 & 0 & 0 & \ldots & 0 & \omega_{j}
\end{array}\right], \quad B_{j}:=\left[\begin{array}{c}
0 \\
\vdots \\
0 \\
1
\end{array}\right], \quad C_{j}:=\left[\begin{array}{lll}
r_{j m_{j}} & \cdots & r_{j 1}
\end{array}\right],
$$

where each column vector $B_{j}$ has $m_{j}$ components and each $A_{j}$ has size $m_{j} \times m_{j}$. Note that we can write (3.2) as

$$
\Pi R(k)=-\mathrm{i}\left[\begin{array}{lll}
C_{1} & \cdots & C_{M}
\end{array}\right]\left[\begin{array}{cccc}
\left(k-\mathrm{i} A_{1}\right)^{-1} & 0 & \ldots & 0 \\
0 & \left(k-\mathrm{i} A_{2}\right)^{-1} & \ldots & 0 \\
\vdots & \vdots & \ddots & \vdots \\
0 & 0 & \ldots & \left(k-\mathrm{i} A_{M}\right)^{-1}
\end{array}\right]\left[\begin{array}{c}
B_{1} \\
\vdots \\
B_{M}
\end{array}\right] .
$$


The above expression corresponds to a minimal realization [35] of $\Pi R$. Associated with the bound-state data $\left\{\kappa_{j}, c_{j}\right\}_{j=1}^{N}$, we let

$$
A_{M+j}:=\kappa_{j}, \quad C_{M+j}:=c_{j}, \quad B_{M+j}:=1, \quad j=1, \ldots, N .
$$

Let us also define

$$
A:=\left[\begin{array}{cccc}
A_{1} & 0 & \ldots & 0 \\
0 & A_{2} & \ldots & 0 \\
\vdots & \vdots & \ddots & \vdots \\
0 & 0 & \ldots & A_{M+N}
\end{array}\right], \quad B:=\left[\begin{array}{c}
B_{1} \\
\vdots \\
B_{M+N}
\end{array}\right], \quad C:=\left[\begin{array}{lll}
C_{1} & \ldots & C_{M+N}
\end{array}\right] .
$$

Note that $A$ is a $P \times P$ block square matrix, $B$ is a column $P$-vector and $C$ is a row $P$-vector, where $P$ is the constant given by

$$
P:=N+2 \sum_{j=1}^{n} m_{j}+\sum_{j=2 n+1}^{M} m_{j}
$$

We also note that all the entries in $A, B$ and $C$ are real constants.

\section{Explicit solutions}

In this section, we construct our explicit solutions in terms of the three matrices $A, B$ and $C$. In section 3 , we have described how $A, B, C$ may be related to some scattering data. Let us define

$$
\Gamma(x ; t):=I_{P}+\int_{x}^{\infty} \mathrm{d} z \mathrm{e}^{-z A} B C \mathrm{e}^{-z A} \mathrm{e}^{8 t A^{3}+2 \eta A t},
$$

where $I_{P}$ is the $P \times P$ unit matrix. Our main result is that the quantity $u(x, t)$ given as

$$
u(x, t)=-2 \frac{\partial}{\partial x}\left[\frac{\frac{\partial}{\partial x} \operatorname{det} \Gamma(x ; t)}{\operatorname{det} \Gamma(x ; t)}\right]
$$

is a solution to $(1.1)$ as long as $\operatorname{det} \Gamma(x ; t)>0$ or, equivalently, as long as the matrix $\Gamma(x ; t)$ is invertible. It is known [11] that $\operatorname{det} \Gamma(x ; 0)>0$ for $x \in[0,+\infty)$. As seen from (4.1), the matrix $\Gamma(x ; t)$ can be explicitly constructed from $A, B$ and $C$, and as argued in section 1 we have det $\Gamma(x ; t)>0$ for all $x \in[0,+\infty)$ and $t \in[0, \tau)$ for some $\tau>0$. There are two possibilities: if $\tau=+\infty$ then the solution $u(x, t)$ given in (4.2) is a global-in-time solution to (1.1); otherwise, it is a local-in-time solution.

The proof that (4.2) satisfies (1.1) when $\Gamma(x ; t)$ is invertible can be outlined as follows. The solution to (1.1) via the inverse scattering transform is obtained as in the diagram

$$
\begin{array}{cc}
\left\{R(k),\left\{\kappa_{j}\right\},\left\{c_{j}\right\}\right\} & \overleftrightarrow{\text { direct scattering }} u(x, 0) \\
\text { time evolution } \downarrow & \downarrow \text { solution to KdV } \\
\left\{R(k) \mathrm{e}^{8 \mathrm{i} k^{3} t-2 \mathrm{i} \eta k t},\left\{\kappa_{j}\right\},\left\{c_{j} \mathrm{e}^{8 \kappa_{j}^{3} t+2 \eta \kappa_{j} t}\right\}\right\} & \stackrel{\text { inverse scattering }}{\longrightarrow} u(x, t)
\end{array}
$$

The inverse scattering step in (4.3) for $x>0$ can be accomplished by solving the time-evolved Marchenko equation [4, 5, 9]:

$K(x, y ; t)+\Omega(x+y ; t)+\int_{x}^{\infty} \mathrm{d} z K(x, z ; t) \Omega(y+z ; t)=0, \quad y>x>0$, 
where the Marchenko kernel $\Omega(y ; t)$ is given by

$$
\Omega(y ; t):=\frac{1}{2 \pi} \int_{-\infty}^{\infty} \mathrm{d} k R(k) \mathrm{e}^{8 \mathrm{i} k^{3} t-2 \mathrm{i} \eta k t+\mathrm{i} k y}+\sum_{j=1}^{N} c_{j} \mathrm{e}^{8 \kappa_{j}^{3} t+2 \eta \kappa_{j} t-\kappa_{j} y} .
$$

If $t=0$ in (4.5) then we can explicitly evaluate $\Omega(y ; 0)$ in terms of $A, B, C$ given in (3.3), and this can be accomplished with the help of the generalized Cauchy integral formula by using a contour integration along the boundary of $\mathbf{C}^{+}$. In general, we cannot evaluate $\Omega(y ; t)$ the same way for all $t>0$, although there are cases when we might be able to do this; for example, if all the eigenvalues of $8 A^{3}+2 \eta A$ have nonpositive real parts, then we might explicitly evaluate $\Omega(y ; t)$ and obtain

$$
\Omega(y ; t)=C \mathrm{e}^{8 t A^{3}+2 \eta A t-y A} B .
$$

It turns out that the evaluation of (4.5) as (4.6) yields (4.9), which is a solution to (1.1) as long as $\Gamma(x ; t)$ is invertible. As discussed in section 1 , this invertibility holds for all $x \in[0,+\infty)$ and $t \in[0, \tau)$ for some $\tau>0$, where the value of $\tau$ depends on the value of $\eta$ and the entries of the constant matrices $A$ and $C$ given in (3.3). We can write $\Omega(x+y ; t)$ as a dot product of a $P$-vector not containing $x$ and a $P$-vector not containing $y$. This separability is easily seen from (4.6) by writing

$$
\Omega(x+y ; t)=C \mathrm{e}^{8 t A^{3}+2 \eta A t-x A} \mathrm{e}^{-y A} B,
$$

where $C \mathrm{e}^{8 t A^{3}+2 \eta A t-x A}$ is a row $P$-vector and $\mathrm{e}^{-y A} B$ is a column $P$-vector. The degeneracy of the kernel $\Omega(y ; t)$ allows us to solve (4.4) explicitly by algebraic means. In fact, its explicit solution is given by

$$
K(x, y ; t)=-C \mathrm{e}^{8 t A^{3}+2 \eta A t-A x} \Gamma(x ; t)^{-1} \mathrm{e}^{-y A} B,
$$

where $\Gamma(x ; t)$ is the matrix in (4.1). Finally, the time-evolved potential $u(x, t)$, which is also a solution to (1.1), is obtained from $(4.8)$ via $[4,5,9]$

$$
u(x, t)=-2 \frac{\partial K(x, x ; t)}{\partial x}
$$

leading to

$$
u(x, t)=2 \frac{\partial}{\partial x}\left[C \mathrm{e}^{8 t A^{3}+2 \eta A t-A x} \Gamma(x ; t)^{-1} \mathrm{e}^{-x A} B\right] .
$$

From (4.1) and (4.9) we obtain

$$
u(x, t)=-2 \frac{\partial}{\partial x} \operatorname{tr}\left[\Gamma(x ; t)^{-1} \frac{\partial}{\partial x} \Gamma(x ; t)\right],
$$

where we have used the fact that in evaluating the trace of a product of two matrices we can change the order in the product. Using theorem 7.3 on p 38 of [36], we can write (4.10) also as (1.2) or (4.2).

As indicated in section 1 , it is somehow surprising that any set of arbitrary choices for $A, B, C$ in (4.1) and (4.9) yields a formal solution to (1.1). It can independently and directly be verified that $u(x, t)$ given in (4.9) is a solution to (1.1) in a region in the $x t$-plane as long as $\Gamma(x ; t)$ exists and is invertible in that region. The verification of this can be achieved in a straightforward way by taking the appropriate derivatives of the right-hand side of (4.9) and substituting them in the left-hand side of (1.1). 


\section{An example}

We will now illustrate our method by an explicit example. Consider the scattering data with no bound states and

$$
\Pi R(k)=\frac{-2 \mathrm{i} \epsilon(k-\mathrm{i} / 2)-\sqrt{3} \gamma}{(k-\mathrm{i} / 2)^{2}-3 / 4},
$$

where $\epsilon$ and $\gamma$ are some positive constants. Using (5.1) in (3.3) we obtain

$$
A=\left[\begin{array}{cc}
1 / 2 & -\sqrt{3} / 2 \\
\sqrt{3} / 2 & 1 / 2
\end{array}\right], \quad B=\left[\begin{array}{l}
0 \\
1
\end{array}\right], \quad C=2\left[\begin{array}{ll}
\gamma & \epsilon
\end{array}\right] .
$$

Alternatively, we can start with $A, B, C$ given in (5.2) without even knowing that they may be related to some scattering data. The use of (5.2) in (4.1) results in

$$
\begin{aligned}
\operatorname{det} \Gamma(x ; t)= & 1-\frac{3}{4}\left(\epsilon^{2}+\gamma^{2}\right) \mathrm{e}^{2(\eta-8) t-2 x} \\
& +\frac{1}{2} \mathrm{e}^{(\eta-8) t-x}[(\sqrt{3} \epsilon-\gamma) \sin (\sqrt{3} \eta t-\sqrt{3} x)+(\epsilon+\sqrt{3} \gamma) \cos (\sqrt{3} \eta t-\sqrt{3} x)] .
\end{aligned}
$$

Note that $\operatorname{det} \Gamma(x ; t)>0$ for all $x, t \geqslant 0$ if $\left(\epsilon^{2}+\gamma^{2}\right)<4 / 9$ and $0 \leqslant \eta \leqslant 8$. It can directly be verified that $u(x, t)$ obtained as in (4.2) with $\operatorname{det} \Gamma(x ; t)$ given in (5.3) solves (1.1) and hence it is a global-in-time solution. Not imposing such restrictions on $\epsilon, \gamma$ and $\eta$, we still obtain solutions to (1.1), which may however be only locally well behaved or may even have singularities.

For example, by choosing $\epsilon=\gamma=1 / 2$ and $\eta=1$, we obtain the explicit solution to $(1.1)$ in the form

$$
u(x, t)=\frac{\phi(x, t)}{\left[1-\frac{3}{8} \mathrm{e}^{-2(x+7 t)}+\frac{1}{\sqrt{2}} \mathrm{e}^{-(x+7 t)} \cos (\sqrt{3}(x-t)+\pi / 12)\right]^{2}},
$$

where we have defined

$$
\begin{gathered}
\phi(x, t):=6 \mathrm{e}^{-2(x+7 t)}-4 \sqrt{2} \mathrm{e}^{-(x+7 t)} \sin (\sqrt{3}(x-t)-\pi / 12) \\
-\frac{3}{\sqrt{2}} \mathrm{e}^{-3(x+7 t)} \sin (\sqrt{3}(x-t)+\pi / 4) .
\end{gathered}
$$

This solution is valid for all $x \in[0,+\infty)$ and $t \in[0,+\infty)$, and its Mathematica animation is available [37].

Adding bound states in our example results in global-in-time solutions containing solitons. For example, by choosing

$$
A=\left[\begin{array}{ccc}
1 / 2 & -\sqrt{3} / 2 & 0 \\
\sqrt{3} / 2 & 1 / 2 & 0 \\
0 & 0 & \kappa_{1}
\end{array}\right], \quad B=\left[\begin{array}{l}
0 \\
1 \\
1
\end{array}\right], \quad C=\left[\begin{array}{lll}
2 \gamma & 2 \epsilon & c_{1}
\end{array}\right]
$$

we get another explicit solution to (1.1) valid for all $x \in[0,+\infty)$ and $t \in[0,+\infty)$. An explicit display of $u(x, t)$ corresponding to (5.4) is available in a Mathematica file, but it takes many pages to display it; its animation with $\epsilon=1 / 2, \gamma=1 / 2, \eta=1, \kappa_{1}=2$ and $c_{1}=3$ is also available in the same Mathematica file [37]. The explicit global-in-time solution and its Mathematica animation are also available [37] in a Mathematica file for the choices

$$
A=\left[\begin{array}{cccc}
1 / 2 & -\sqrt{3} / 2 & 0 & 0 \\
\sqrt{3} / 2 & 1 / 2 & 0 & 0 \\
0 & 0 & \kappa_{1} & 0 \\
0 & 0 & 0 & \kappa_{2}
\end{array}\right], \quad B=\left[\begin{array}{l}
0 \\
1 \\
1 \\
1
\end{array}\right], \quad C=\left[\begin{array}{llll}
2 \gamma & 2 \epsilon & c_{1} & c_{2}
\end{array}\right] \text {, }
$$

which contains two solitons. 


\section{Acknowledgments}

The research leading to this paper was supported in part by the National Science Foundation under grant DMS-0610494, the Italian Ministry of Education and Research (MIUR) under COFIN grant no 2004015437 and INdAM-GNCS.

\section{References}

[1] Bona J L, Sun S M and Zhang B Y 2002 A non-homogeneous boundary-value problem for the Korteweg-de Vries equation in a quarter plane Trans. Am. Math. Soc. 354 427-90

[2] Fokas A S 2002 Integrable nonlinear evolution equations on the half-line Commun. Math. Phys. 230 1-39

[3] Zabusky N J and Kruskal M D 1965 Interaction of 'solitons' in a collisionless plasma and the recurrence of initial states Phys. Rev. Lett. 15 240-3

[4] Ablowitz M J and Segur H 1981 Solitons and the Inverse Scattering Transform (Philadelphia, PA: SIAM)

[5] Ablowitz M J and Clarkson P A 1991 Solitons, Nonlinear Evolution Equations and Inverse Scattering (London: Cambridge University Press)

[6] Gardner C S, Greene J M, Kruskal M D and Miura R M 1967 Method for solving the Korteweg-de Vries equation Phys. Rev. Lett. 19 1095-7

[7] Marchenko V A 1988 Nonlinear Equations and Operator Algebras (Dordrecht: Reidel)

[8] Matveev V B and Salle M A 1991 Darboux Transformations and Solitons (Berlin: Springer)

[9] Aktosun T 2005 Solitons and inverse scattering transform Mathematical Studies in Nonlinear Wave Propagation (Contemp. Math. vol 379) ed D P Clemence and G Tang (Providence, RI: American Mathematical Society) pp 47-62

[10] Colliander J E and Kenig C E 2002 The generalized Korteweg-de Vries equation on the half line Commun. Partial Diff. Eqns 27 2187-266

[11] Aktosun T, Klaus M and van der Mee C 1992 Explicit Wiener-Hopf factorization for certain nonrational matrix functions Integral Eqns Operator Theory 15 879-900

[12] Wahlquist H D and Estabrook F B 1975 Prolongation structures and nonlinear evolution equations J. Math. Phys. 16 1-7

[13] Konopel'chenko B G 1990 On exact solutions of nonlinear integrable equations via integral linearising transforms and generalised Bäcklund-Darboux transformations J. Phys. A: Math. Gen. 23 3761-8

[14] Huang N N 1992 Darboux transformations for the Korteweg-de Vries equation J. Phys. A: Math. Gen. 25 469-83

[15] Freeman N C and Nimmo J J C 1983 Soliton solutions of the Korteweg-de Vries and Kadomtsev-Petviashvili equations: the Wronskian technique Phys. Lett. A 95 1-3

[16] Matveev V B 1992 Generalized Wronskian formula for solutions of the KdV equations: first applications Phys. Lett. A $166205-8$

[17] Carl B and Schiebold C 2000 Ein direkter Ansatz zur Untersuchung von Solitonengleichungen Jahresber. Deutsch. Math.-Verein. 102 102-48

[18] Ma W-X and You Y 2005 Solving the Korteweg-de Vries equation by its bilinear form: Wronskian solutions Trans. Am. Math. Soc. 357 1753-78

[19] Hirota R 2004 The Direct Method in Soliton Theory (Cambridge: Cambridge University Press)

[20] Treibich A and Verdier J L 1990 Solitons elliptiques The Grothendieck Festschrift vol III (Progr. Math. vol 88) (Boston, MA: Birkhäuser Boston) pp 437-80

[21] Gesztesy F and Weikard R 1998 Elliptic algebro-geometric solutions of the KdV and AKNS hierarchies-an analytic approach Bull. Am. Math. Soc. (N.S.) 35 271-317

[22] Gesztesy F, Unterkofler K and Weikard R 2006 An explicit characterization of Calogero-Moser systems Trans. Am. Math. Soc. 358 603-56

[23] Ablowitz M J and Satsuma J 1978 Solitons and rational solutions of nonlinear evolution equations J. Math. Phys. 19 2180-6

[24] Arkad'ev V A, Pogrebkov A K and Polivanov M K 1985 Singular solutions of the KdV equation and the inverse scattering method J. Math. Sci. 31 3264-79

[25] Matveev V B 1992 Positon-positon and soliton-positon collisions: KdV case Phys. Lett. A 166 209-12

[26] Kovalyov M 1998 Basic motions of the Korteweg-de Vries equation Nonlinear Anal. 31 599-619

[27] Gohberg I, Kaashoek M A and Sakhnovich A L 1998 Sturm-Liouville systems with rational Weyl functions: explicit formulas and applications Integral Eqns Operator Theory 30 338-77

[28] Jaworski M 1984 Breather-like solutions to the Korteweg-de Vries equation Phys. Lett. A 104 245-7 
[29] Mei J-q and Zhang H-q 2005 New soliton-like and periodic-like solutions for the KdV equation Appl. Math. Comput. 169 589-99

[30] Aktosun T and Klaus M 2001 Inverse theory: problem on the line Scattering ed E R Pike and P C Sabatier (London: Academic) chapter 2.2.4 pp 770-85

[31] Faddeev L D 1967 Properties of the $S$-matrix of the one-dimensional Schrödinger equation Am. Math. Soc. Transl. (Ser. 2) 65 139-66

[32] Deift P and Trubowitz E 1979 Inverse scattering on the line Commun. Pure Appl. Math. 32 121-251

[33] Marchenko V A 1986 Sturm-Liouville Operators and Applications (Basel: Birkhäuser)

[34] Chadan K and Sabatier P C 1989 Inverse Problems in Quantum Scattering Theory 2nd edn (New York: Springer)

[35] Bart H, Gohberg I and Kaashoek M A 1979 Minimal Factorization of Matrix and Operator Functions (Basel: Birkhäuser)

[36] Coddington E A and Levinson N 1955 Theory of Ordinary Differential Equations (New York: McGraw-Hill)

[37] http://omega.uta.edu/ aktosun 